\title{
The effect of third principal stress in the measurement of residual stresses by hole-drilling method
}

\author{
David Halabuk $^{1, a}$ and Tomas Navrat ${ }^{2}$ \\ ${ }^{1}$ Faculty of Mechanical Engineering, Brno University of Technology, Technicka 2896/2, 61669 Brno, Czech \\ Republic
}

\begin{abstract}
One of the most popular and widely used technique for measuring residual stresses is the hole-drilling method. By this method, it is possible to evaluate only biaxial residual stresses located in plane parallel to the surface, but some processes produce a triaxial stress state. For this reason, the evaluation of triaxial stress state by the method used for biaxial state was assessed in this paper. A hole-drilling experiment was simulated by the finite element method for two different stress states. The first stress state considered constant residual stresses in all directions. The second one considered constant residual stresses in a plane parallel to the surface and the residual stress in a direction perpendicular to the surface was equal to zero on the surface and increased with depth. Both states were simulated for various ratios of stress in a direction perpendicular to the stresses in plane. The obtained results show that residual stress in a perpendicular direction affects the evaluation of residual stresses in plane. If the residual stress in the perpendicular direction is high compared to stresses in plane, the error produced by the evaluation of triaxial stress state by the method for biaxial stress state can also be high.
\end{abstract}

\section{Introduction}

Residual stresses are usually created in a component as a consequence of manufacturing, heat treatment or loading and can have positive or negative effects. On the one hand, they can cause a change of the component's shape after machining or cracks (Fig. 1) [1]. On the other hand, they can also suppress the creation of cracks or decrease the total stress in a component during loading. The positive or negative effect depends on a sign and magnitude of residual stresses. For this reason, it is important to know the correct value of residual stresses in components. The hole-drilling method is one of the most popular and widely used techniques for measuring residual stresses near the surface of component. This method is based on drilling a small hole to the measured component which causes a redistribution of residual stresses around it (Fig. 2). After that, relaxed strains are measured by a strain gauge rosette and residual stresses are evaluated from these measured strains [2].

\footnotetext{
${ }^{\mathrm{a}}$ Corresponding author : david.halabuk@vutbr.cz
} 


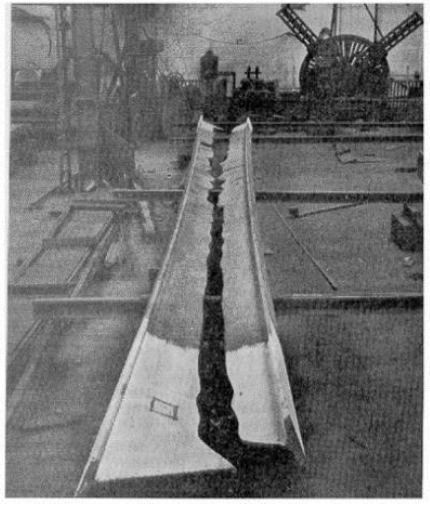

Figure 1. Beam split due to relaxation of residual stresses [1].

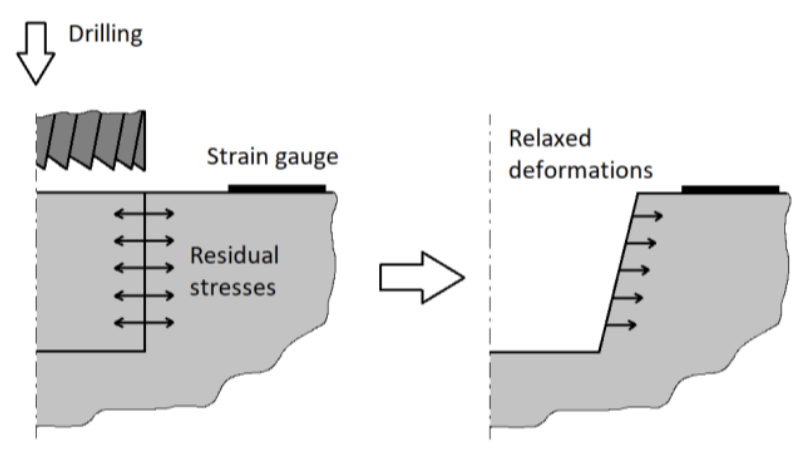

Figure 2. The principle of hole-drilling method.

The accuracy of residual stress evaluation depends on many factors. If the hole is drilled eccentrically, shape or diameter of drilled hole is different from considered ones, or there are extensive plastic deformations the error is produced during evaluation $[3,4]$. It is important to known which factors influence the evaluation of residual stresses and how strong this influence is.

There are several methods for evaluating the measured strains. Some of them are convenient for uniform residual stress distribution in depth direction, others for non-uniform ones. Moreover, some methods deal with eccentrically drilled hole or with plastic deformations which occurred around drilled hole when residual stresses were close enough to yield strength of material before hole drilling. However, all methods are able to evaluate only biaxial stress states when two principal stresses $\left(\sigma_{\mathrm{x}}, \sigma_{\mathrm{y}}\right)$ are located in a plane parallel to the surface of the component. They assume that third stress $\left(\sigma_{z}\right)$ situated in perpendicular direction to the surface is equal to zero or has a negligible effect on the evaluation of residual stresses $\sigma_{x}, \sigma_{y}$.

As shown in $[5,6]$, processing such as shot peening, welding, or quenching can produce triaxial residual stress states. In most cases, residual stress $\sigma_{z}$ is equal to zero at the surface of component but an increase in $\sigma_{z}$ can be significant under the surface and thus $\sigma_{z}$ can affect the evaluation of residual stresses. It is a problem to evaluate triaxial residual stress states because strain gauge rosettes do not provide necessary amount of information. If we want to deal with residual stress in a direction perpendicular to the surface, we also need to measure a strian or a displacement in this direction. However, in practical applications this displacement is hard to measure. Wern et al. [7] modified an integral method, usually used for biaxial states, for the evaluation of triaxial residual states by additional information about the displacement in the direction perpendicular to the surface. However, there is no known publication in which this method is applied in practice. Moreover, the influence of residual stress $\sigma_{z}$ on the evaluation of residual stresses in plane parallel to the surface is also unknown. Since in practical application, as mentioned above, triaxial residual stress state can be created it is important to know this influence. This is the reason why the evaluation of triaxial residual stress states by a method taking into account biaxial ones and the calculation of errors which are produced by this procedure are presented in this paper.

\section{Methods}

A hole drilling experiment was simulated by the finite element method. For this purpose, the ANSYS software was used. By simulating the hole drilling experiment, it is possible to exclude the uncertainty of material characteristics, measured residual stresses, technological factors or errors caused by measurement process. 
A model of thick solid body with a uniform mesh around the drilled hole was created. Linear elements were used for the mesh; the total number of elements was over 2.5 million. Drilling process was simulated by a sequential removal of layers of mesh in place where a hole should be created. Fig. 3 depicts the finite element mesh of a model with a drilled hole.

The linear model of material with an elastic modulus of $210 \mathrm{GPa}$ and Poisson's ratio of 0.3 was used. The drilled hole had a diameter of $2 \mathrm{~mm}$ and a depth of $1 \mathrm{~mm}$. The drilling process was simulated by 0.05 depth increment.

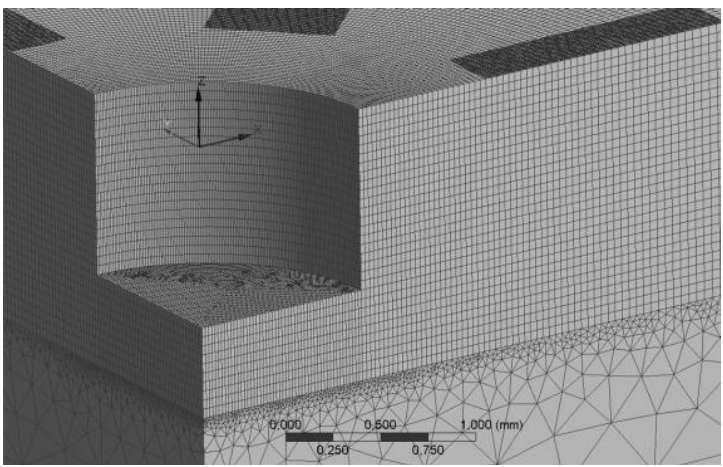

Figure 3. The mesh of finite element method model.

Relaxed strains, after removing the 'drilled-out' elements, were obtained from averaging nodal strains on surface bodies which represented grids of a strain gage rosette. These surface bodies had dimensions of a real strain gauge rosette 1-RY61-1,5/120S (HBM) and were bonded to the surface of measured solid body. Because of symmetry, only a quarter of the whole model was simulated. Two residual stress states were simulated (Fig. 4).

a)

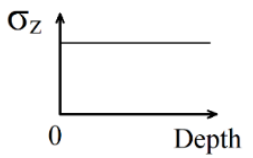

b)

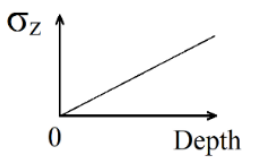

c)

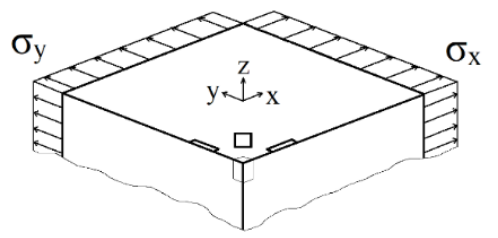

Figure 4. Stress state with a uniform residual stress $\sigma_{z}$ in depth direction (a) and stress state with $\sigma_{z}$ equal to zero on the surface and a linear increase with depth (b); stresses $\sigma_{\mathrm{x}}$ and $\sigma_{\mathrm{y}}$ are uniform for both stress states (c).

The first stress state considers a uniform residual stress $\sigma_{z}$ whose value differs from 0 to $250 \mathrm{MPa}$ for analyses. Residual stresses $\sigma_{\mathrm{x}}$ and $\sigma_{\mathrm{y}}$ were also uniform with a constant value of $500 \mathrm{MPa}$ for all analyses. The stress state was induced by a command which inputs the values of stresses into every element of the mesh at the beginning of the analysis. For the first state, seven analyses with the $\sigma_{z}$ to $\sigma_{\mathrm{x}}$ ratio equal to $0,0.05,0.1,0.2,0.3,0.4$ and 0.5 were calculated.

The second stress state had $\sigma_{z}$ equal to zero on the surface and its value increased with depth. Values of residual stresses for the second stress state depending on the depth direction are shown in Fig. 5. Individual analyses for this state are labelled by the $\sigma_{z}$ to $\sigma_{x}$ ratio in the depth of $1 \mathrm{~mm}$. This stress state is closer to reality than the first one because $\sigma_{z}$ is usually equal to zero on the surface. Residual stresses $\sigma_{\mathrm{x}}, \sigma_{\mathrm{y}}$ are, as for the first stress state, uniform with the value of $500 \mathrm{MPa}$. This stress state was induced by a non-uniform temperature distribution in combination with volume forces. 


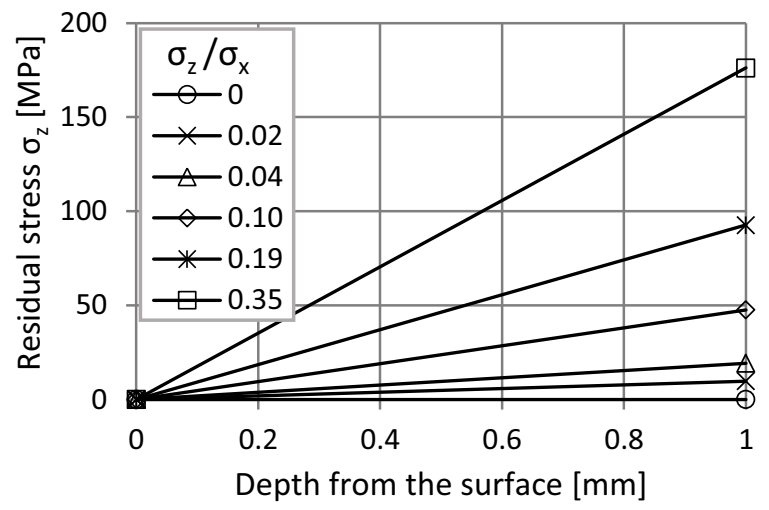

Figure 5. Values of residual stresses $\sigma_{\mathrm{z}}$ depending on the depth direction for the second stress state.

Strains obtained from simulations were evaluated by the EVAL-7 software and residual stresses were calculated by the ASTM E837-13 Extended Uniform calculation method. Thus calculated residual stresses were compared to the simulated ones and a relative error was calculated as follows.

$$
\delta=\frac{\sigma_{e}-\sigma_{s}}{\sigma_{s}} \cdot 100[\%]
$$

where $\delta$ is the relative error, $\sigma_{e}$ and $\sigma_{s}$ are evaluated and simulated residual stresses in the $x$-direction respectively. Because simulated stresses in the $x$ and $y$-directions were the same, also relative error was the same in these directions except for a negligible numerical error. For this reason, relative errors were plotted only for residual stress $\sigma_{\mathrm{x}}$.

\section{Results and discussion}

Fig. 6 illustrates the distribution of normal strain in the $x$-direction after hole drilling for the first stress state and $\sigma_{z} / \sigma_{x}=0$ (a) and $\sigma_{z} / \sigma_{x}=0.5(b)$. It can be noticed that the distribution in component is slightly different between states with different $\sigma_{z}$ to $\sigma_{x}$ ratios, but more importantly, the difference on the surface in the area of strain gauge grid situated in the $x$-direction can be observed. If there is a difference of strains on the surface of the component, the value of strain measured by the strain gauge rosette is also different, which affects the evaluation of residual stresses.

a)

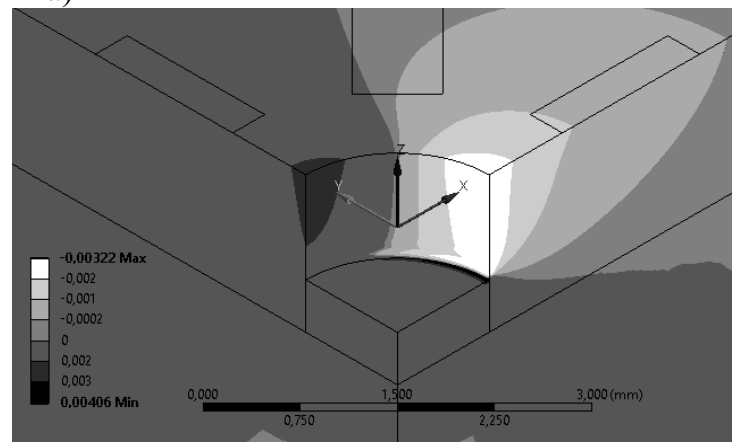

b)

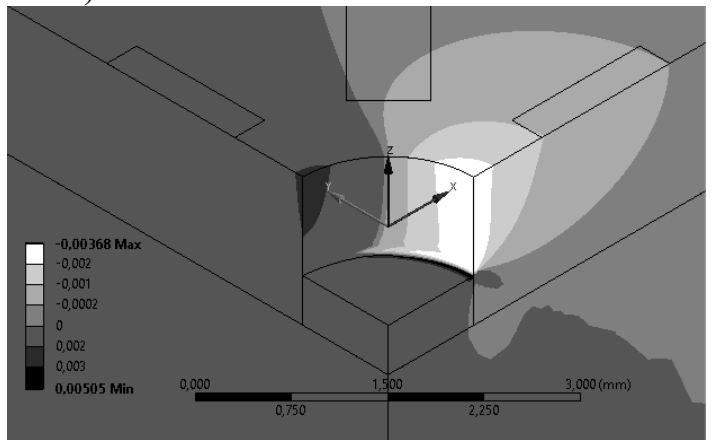

Figure 6. The strain distribution in the $x$-direction for the first stress state with $\sigma_{z} / \sigma_{x}=0$ (a) and $\sigma_{z} / \sigma_{x}=0.5$ (b).

Values of the measured strains for various $\sigma_{z}$ to $\sigma_{x}$ ratios are plotted in Fig. 7. The presence of residual stress $\sigma_{\mathrm{z}}$ caused that the strains measured by the strain gauge rosette are higher for all depth 
increments than those with $\sigma_{\mathrm{z}}$ equal to zero. The higher the $\sigma_{\mathrm{z}}$ to $\sigma_{\mathrm{x}}$ ratio, the higher the measured strains.

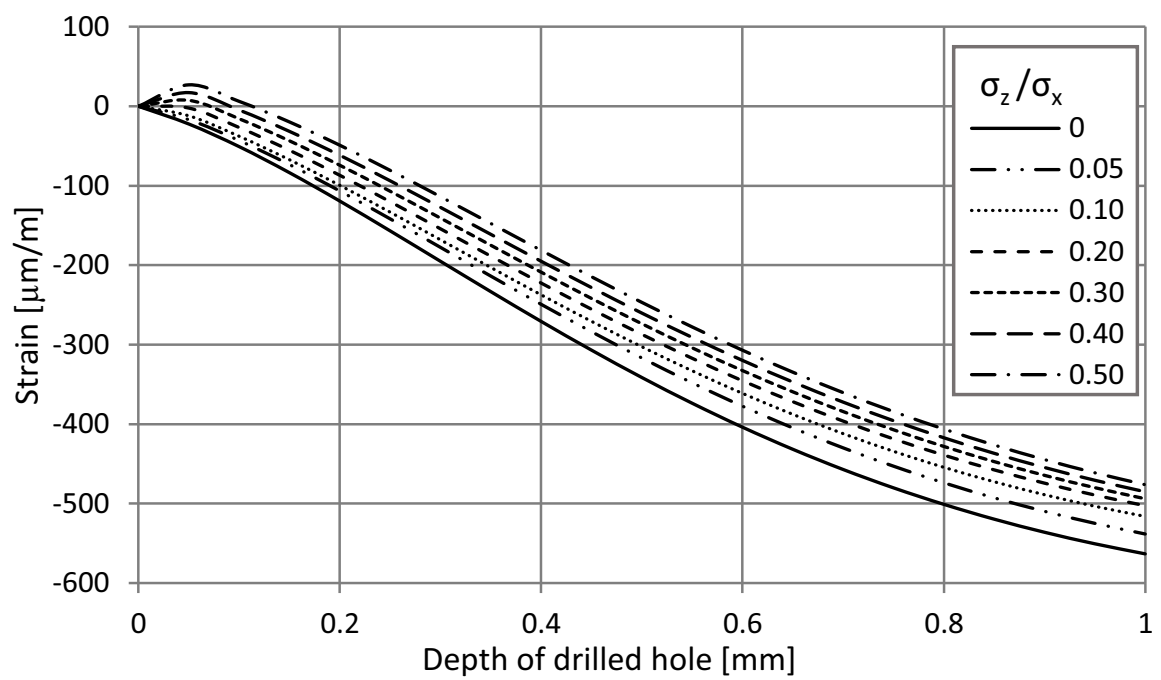

Figure 7. Strains obtained from strain gauge grid situated in $x$-direction for state with uniform $\sigma_{z}$ in depth direction

For a state with non-uniform residual stress $\sigma_{z}$ there is also upward shift of curves (Fig. 8). However, in this case, due to linear increase of $\sigma_{\mathrm{z}}$ from zero on the surface, the shift starts from zero in the zero depth and increase with depth. In addition, the shift for same $\sigma_{\mathrm{z}}$ to $\sigma_{\mathrm{x}}$ ratios in the depth of $1 \mathrm{~mm}$ is lower for this stress state than for the stress state with uniform $\sigma_{\mathrm{z}}$.

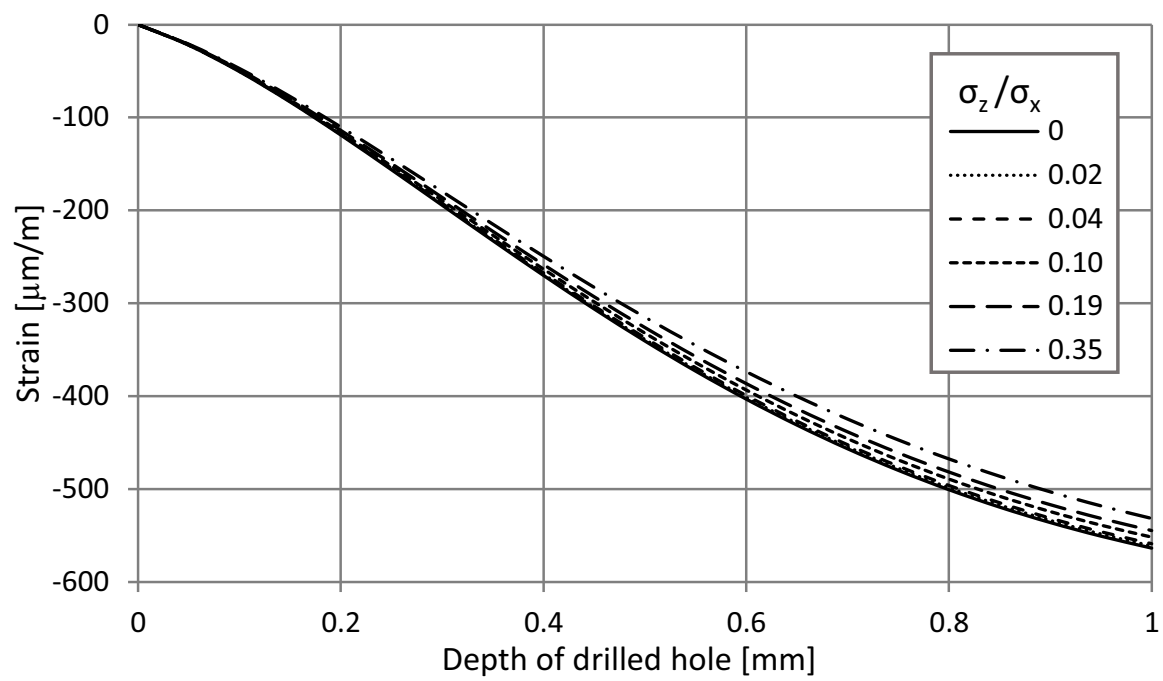

Figure 8. Strains obtained from strain gauge grid situated in $x$-direction for state with $\sigma_{z}$ equal to zero on the surface and increasing with increasing depth

Relative errors of evaluated residual stresses depending on the $\sigma_{\mathrm{z}}$ to $\sigma_{\mathrm{x}}$ ratio for a state with uniform and non-uniform residual stresses $\sigma_{z}$ are illustrated in Fig. 9. Higher values of measured strains for all depth increments for the stress state with a uniform residual stress $\sigma_{z}$ caused that relative errors are high already for small $\sigma_{z}$ to $\sigma_{x}$ ratio. For higher $\sigma_{z}$ to $\sigma_{x}$ ratios a relative error is high and the presence of $\sigma_{\mathrm{z}}$ has a significant influence on the evaluation of residual stresses. If $\sigma_{\mathrm{z}}$ is not constant 
with depth, but increases linearly from zero on the surface, the values of relative errors are considerably lower. For small $\sigma_{\mathrm{z}}$ to $\sigma_{\mathrm{x}}$ ratios the effect of $\sigma_{\mathrm{z}}$ on the evaluation of residual stresses is negligible. However, if $\sigma_{\mathrm{z}}$ is high enough compared to stresses in the plane parallel to the surface, it could negatively affect the evaluation of residual stresses.

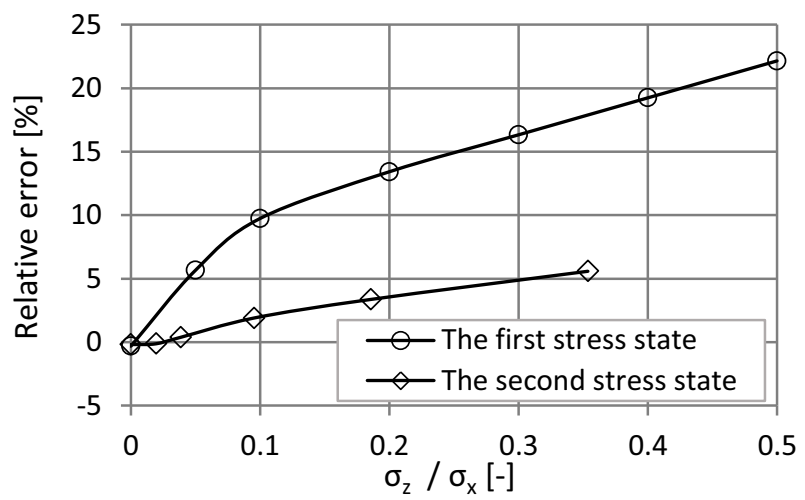

Figure 9. Relative error of evaluated residual stresses for the stress state with uniform $\sigma_{\mathrm{z}}$ (the first stress state) and $\sigma_{z}$ equal to zero on the surface and increasing with increasing depth (the second stress state).

\section{Conclusion}

The presence of residual stresses in the direction perpendicular to the surface causes that strains measured by the strain gauge rosette are different than strains in biaxial stress state. For this reason, the evaluation of triaxial stress states by a method used for biaxial stress states produces additional errors. The value of these errors depends on the $\sigma_{\mathrm{z}}$ to $\sigma_{\mathrm{x}}$ ratio. Simulations of the state with a uniform residual stress $\sigma_{z}$ show that the relative error of evaluated stresses could be high already for small $\sigma_{z}$ to $\sigma_{\mathrm{x}}$ ratio. However, in practice, a state with residual stress $\sigma_{\mathrm{z}}$ equal to zero on the surface and with an increase in the depth direction is more realistic. This state produces considerably lower relative errors and for small ratios the relative error is negligible. Nevertheless, if residual stress $\sigma_{z}$ is high compared to residual stresses $\sigma_{\mathrm{x}}$ or $\sigma_{\mathrm{y}}$, the relative errors of evaluated residual stresses in the plane parallel to the surface can increase to significant values. The value of the $\sigma_{z}$ to $\sigma_{x}$ ratio which is acceptable depends on the required accuracy of residual stress evaluation.

\section{Acknowledgment}

This work is an output of projects FV 18 - 18 and NETME CENTRE PLUS (LO1202) created with financial support from the Ministry of Education, Youth and Sports under the „National Sustainability Programme I“.

\section{References}

1. F. Campus, Effects of residual stresses on the behaviour of structures (1954)

2. G. S. Schajer, Practical Residual Stress Measurement Methods (John Wiley, 2013)

3. D. Halabuk and T. Navrat, EAN 2017 - 55th Conference on Experimental Stress Analysis 2017, 814 (2017)

4. T. Navrat, D. Halabuk, M. Vlk, P. Vosynek, Defect Diffus. Forum 382, 208-212 (2018)

5. G.A. Webster and A.N. Ezeilo, Int. J. Fatigue 23, 375-383 (2001)

6. S. Hossain, C.E. Truman, D.J. Smith, M.R. Daymond, Int. J. Mech. Sci. 43, 235-243 (2006)

7. H. Wern, R.Cavelius, D. Schläfer, Strain 33, 39-46 (1997) 